Quim. Nova, Vol. 37, No. 1, 44-49, 2014

\title{
FLOCULAÇÃO DE Chlorella sp. PRODUZIDA EM CONCENTRADO DE DESSALINIZAÇÃO E ESTUDO DE MÉTODO DE EXTRAÇÃO DE LIPÍDEOS INTRACELULARES
}

\author{
Luiz Rodrigo Ito Morioka, Ângelo Paggi Matos*, Gisele Olivo e Ernani Sebastião Sant’Anna \\ Departamento de Ciência e Tecnologia de Alimentos, Centro de Ciências Agrárias, Universidade Federal de Santa Catarina, Rodovia \\ Admar Gonzaga, 1346 - Itacorubi, 88034-001 Florianópolis - SC, Brasil
}

Recebido em 14/03/2013; aceito em 12/08/2013; publicado na web em 02/09/2013

\begin{abstract}
EVALUATION OF FLOCCULATION AND LIPID EXTRACTION OF Chlorella sp. CULTIVATED IN CONCENTRATED DESALINATION. Chlorella sp. was used to assess algal lipid production with concentrated desalination. In order to investigate the action of the flocculating agent calcium chloride and pH, a Box-Behnken Design and a Central Composite Design (CCD) were carried out. Also, Soxhlet and Supercritical Fluid Extraction (SFE), with and without sonication lipid extraction methods, were examined. The optimal flocculation conditions were $\mathrm{pH} 10.0$ and $2.0 \mathrm{~g} / \mathrm{L}$ of calcium chloride concentration. The highest lipid content of Chlorella sp. was obtained using the Soxhlet extraction method. The most abundant fatty acid extracted by Soxhlet and SFE, with and without sonication, was palmitic acid, whose proportions were $57.4 \%, 35.3 \%$ and $25.5 \%$, respectively.
\end{abstract}

Keywords: concentrated desalination; flocculation; microalga oil.

\section{INTRODUÇÃO}

O Brasil se encontra em uma posição privilegiada para assumir a liderança no aproveitamento integral das biomassas pelo fato de possuir intensa radiação solar, água em abundância, diversidade de clima e pioneirismo na produção de biocombustíveis em larga escala, com destaque na obtenção de bioetanol a partir de cana de açúcar. ${ }^{1}$ As microalgas despontam como um novo recurso renovável com potencialidades diversas em termos de biomassa, biocombustíveis e bioprodutos. ${ }^{2,3}$ Este interesse no cultivo de microalgas, em grande parte, é devido ao rápido crescimento e tolerância a ambientes extremos, bem como por serem facilmente cultivadas em águas salobras e/ou águas residuais. ${ }^{4,5}$ Por exemplo, o principal método utilizado para obtenção de água potável na região nordeste do Brasil é a purificação das águas subterrâneas, na maioria dos casos salobra, pelo processo de dessalinização por osmose reversa. No entanto, a dessalinização de águas salobras produz um concentrado com elevado teor de sais minerais, na qual o tratamento inadequado pode causar problemas ambientais como a salinização do solo e consequentemente infertilidade da terra. ${ }^{6}$ Assim, é geralmente reconhecido que as microalgas desempenham um papel importante na auto-purificação de águas residuais. ${ }^{4,7}$ Desta forma, o cultivo de microalgas em concentrado de dessalinização é uma forma eficiente de remoção de nutrientes. As microalgas assimilam os nutrientes existentes no concentrado e os incorpora em sua biomassa, gerando quantidades significativas de óleo. Ainda, pode-se obter uma biomassa rica em lipídeos e outros compostos nutracêuticos. ${ }^{8}$

O potencial das microalgas em comparação com as plantas para produção de biocombustíveis está relacionado com o alto teor de lipídeos produzido por estes micro-organismos. ${ }^{9}$ Apesar da potencialidade das microalgas como matéria-prima para biocombustíveis, a coleta de algas continua a ser um grande obstáculo econômico, com um custo estimado em $18,5 \%$ dos custos totais de produção. ${ }^{10}$ Os desafios da coleta estão associados à natureza microscópica das algas (tamanho entre 5-20 $\mu \mathrm{m}$ de diâmetro) e à baixa produtividade. ${ }^{11}$ Por conseguinte, a coleta da biomassa de algas é uma importante área de pesquisa a fim de desenvolver um processo econômico e competitivo para a produção de biocombustíveis. Técnicas comuns e primárias para a concentração

\footnotetext{
*e-mail: angelosotam@gmail.com
}

e coleta da biomassa microalgal tem sido realizadas por centrifugação, floculação seguida por sedimentação ou flotação. ${ }^{12}$ Neste estudo será avaliado o processo de floculação-sedimentação de Chlorella sp., por meio da adição de aditivos floculantes - cloreto de cálcio $\left(\mathrm{CaCl}_{2}\right)$. Do mesmo modo, para facilitar a floculação, o $\mathrm{pH}$ do meio de cultivo pode ser ajustado a fim de induzir um fenômeno conhecido como autofloculação. ${ }^{13}$ Desta forma, a determinação da concentração do agente floculante (cloreto de cálcio) e indução do $\mathrm{pH}$ foi otimizada utilizando a metodologia de superfície de resposta (MSR), uma ferramenta estatística que foi anteriormente proposta por Yang et al. ${ }^{14}$

Embora a extração lipídica nas microalgas seja relativamente fácil de ser executada, são usados vários processos para aumentar a eficiência e o rendimento, com baixos custos. O óleo pode ser extraído por processos químicos nos quais solventes como benzeno, éter ou hexano são usados para promover a extração de lipídeos. O uso de enzimas é outro método de extração eficaz, que torna o fracionamento do óleo mais fácil. Além destes métodos, o tradicional método de sonicação (ultrassom) também é utilizado para o rompimento da parede celular das algas. ${ }^{2,15,16}$ Um método bastante inovador é a extração por fluido supercrítico (EFS), onde o gás $\mathrm{CO}_{2}$ sofre pressão e aquecimento, adquirindo ambas propriedades de líquido e gás. Este fluído liquefeito atua como solvente, extraindo o óleo da matéria-prima. Neste processo é possível capturar mais energia da célula lipídica, favorecendo a conversão, sendo substancialmente melhor quando comparado ao processo convencional. ${ }^{17}$

Os objetivos deste trabalho foram cultivar a microalga Chlorella sp. Em um meio à base de concentrado de dessalinização; otimizar a auto-floculação da biomassa microalgal de Chlorella sp. por meio da metodologia de superfície de resposta; e comparar o teor de lipídios e o perfil dos ácidos graxos da microalga utilizando técnicas de extração dos lipídeos da biomassa pelo método convencional (soxhlet) e extração por fluido supercrítico (EFS) com e sem o rompimento da parede celular (sonicação).

\section{PARTE EXPERIMENTAL}

\section{Concentrado de dessalinização}

Amostras do concentrado de dessalinização foram coletadas em 
uma planta de dessalinização de água salobra, localizada no município de São João do Cariri, Paraíba, Brasil. As amostras foram armazenadas em recipientes plásticos de 100 litros em câmara de refrigeração a $-20{ }^{\circ} \mathrm{C}$ no Laboratório de Biotecnologia de Alimentos (BIOTEC) da Universidade Federal de Santa Catarina (UFSC). Características físico-químicas do concentrado de dessalinização (Tabela 1) foram determinadas de acordo com a metodologia descrita por APHA. ${ }^{18}$

Tabela 1. Características físico-químicas do concentrado de dessalinização

\begin{tabular}{lc}
\hline Parâmetro & Valor médio \\
\hline $\mathrm{Cl}(\mathrm{mg} / \mathrm{L})$ & 1691,3 \\
$\mathrm{Ca}(\mathrm{mg} / \mathrm{L})$ & 126,5 \\
$\mathrm{Fe}(\mathrm{mg} / \mathrm{L})$ & 0,13 \\
$\mathrm{~K}(\mathrm{mg} / \mathrm{L})$ & 47,0 \\
$\mathrm{Mg}(\mathrm{mg} / \mathrm{L})$ & 4,74 \\
$\mathrm{Na}(\mathrm{mg} / \mathrm{L})$ & 987,5 \\
$\mathrm{NH}{ }_{4}^{+}(\mathrm{mg} / \mathrm{L})$ & 1,35 \\
Sulfatos $(\mathrm{mg} / \mathrm{L})$ & 138,0 \\
Fósforos totais $\left(\mathrm{PO}_{4}{ }^{3-} \mathrm{mg} / \mathrm{L}\right)$ & 0,70 \\
Dureza total $\left(\mathrm{CaCO}_{3}, \mathrm{mg} / \mathrm{L}\right)$ & 985,2 \\
TDS (mg/L) & 2190,5 \\
Nitrogênio total $\left(\mathrm{mg}^{2} / \mathrm{L}\right)$ & 30,0 \\
Condutividade $\left(\mu \mathrm{SS}_{\mathrm{cm}}\right)$ & 4875,0 \\
pH & 8,11 \\
\hline
\end{tabular}

Nota $:$ TDS = Sólidos Totais Dissolvidos.

\section{Micro-organismo e preparação do inóculo}

Uma cepa selvagem, chamada TU1, foi obtida em tanques de cultivo de peixes contendo concentrado de dessalinização em São João do Cariri, Paraíba, Brasil. A estirpe foi purificada em meio de cultura Bold Basal Medium (BBM) solidificado com ágar a 1,5\%. A cepa (TU1) cresceu bem em meio BBM e foi identificada morfologicamente como espécie Chlorella, renomeada como Chlorella sp. A microalga foi mantida em Bold Basal Medium (BBM) modificado ${ }^{19}$ contendo os seguintes componentes (por litro): $0.075 \mathrm{~g}$ $\mathrm{K}_{2} \mathrm{HPO}_{4}, 0.014 \mathrm{~g} \mathrm{KH}_{2} \mathrm{PO}, 0.075 \mathrm{~g} \mathrm{MgSO}_{4} .7 \mathrm{H}_{2} \mathrm{O}, 0.09 \mathrm{~g} \mathrm{NaNO}_{3}$, $0.025 \mathrm{~g} \mathrm{CaCl}_{3} .2 \mathrm{H}_{2} \mathrm{O}, 0.025 \mathrm{~g} \mathrm{NaCl}, 0.05 \mathrm{~g} \mathrm{EDTA}^{-N_{4}}, 0.00498 \mathrm{~g}$ $\mathrm{FeSO}_{4} .7 \mathrm{H}_{2} \mathrm{O}, 0.01142 \mathrm{~g} \mathrm{H}_{3} \mathrm{BO}_{3}, 0.232 \mathrm{mg} \mathrm{MnCl}_{2} .4 \mathrm{H}_{2} \mathrm{O}, 1.41 \mathrm{mg}$ $\mathrm{ZnSO}_{4} .7 \mathrm{H}_{2} \mathrm{O}, 0.252 \mathrm{mg} \mathrm{CuSO} .5 \mathrm{H}_{2} \mathrm{O}, 0.192 \mathrm{mg} \mathrm{NaMoO} .5 \mathrm{H}_{2} \mathrm{O}$, $0.080 \mathrm{mg} \mathrm{CoCl} \cdot 6 \mathrm{H}_{2} \mathrm{O}, 10 \mathrm{~g}$ de extrato de levedura e $250 \mathrm{~mL}$ de concentrado de dessalinização.

A microalga cultivada até a fase exponencial foi utilizada como inóculo, que foi transferido em um volume correspondente a $10 \%$ (v/v) para biorreatores cônicos invertidos com capacidade de 4.000 $\mathrm{mL}$, contendo $3.500 \mathrm{~mL}$ de solução BBM modificado. Os cultivos foram mantidos sob aeração constante, temperatura de $27^{\circ} \mathrm{C}$, sob iluminação de 10 klux fornecida por lâmpadas fluorescentes de $40 \mathrm{~W}$, com fotoperíodo de 12-h claro/12-h escuro durante 12 dias de cultivo.

\section{Planejamento experimental}

O estudo dos efeitos e de otimização das condições do volume de sedimentação por meio da floculação da biomassa de Chlorella sp. foi realizado mediante a metodologia de superfície de resposta e consistiu em duas etapas. Na primeira etapa foi utilizado um delineamento estatístico de Box-Behnken com 2 fatores ( $\mathrm{pH}$ e cloreto de cálcio) e 3 níveis $(-1,0,+1) .{ }^{20}$ Os níveis reais dos fatores independentes foram
0,5; 1,5 e 2,5 (g/L) para o cloreto de cálcio $\left(\mathrm{CaCl}_{2}\right)$ e 7,0; 8,0 e 9,0 para o $\mathrm{pH}$ (Tabela 2). Posteriormente, um Delineamento Central Composto (DCC) foi utilizado com uma região experimental de 5 níveis (valores codificados em $-\alpha,-1,0,+1 \mathrm{e}+\alpha$; Tabela 4 ). O ponto central (0) foi replicado 3 vezes para estimativa do erro experimental, totalizando 13 ensaios. Os valores codificados foram transformados em valores reais segundo a Equação 1

$Y=\beta_{0}+\beta_{1} X_{1}+\beta_{2} X_{2}+\beta_{3} X_{3}+\beta_{11} X_{1}^{2}+\beta_{22} X_{2}^{2}+\beta_{33} X_{3}^{2}+\beta_{12} X_{1} X_{3}+$ $\beta_{23} X_{2} X_{3}+\varepsilon$

onde, Y a variável predita, $\beta_{0}$ o intercepto; $\beta_{1}, \beta_{2}, \beta_{3}$ os coeficientes lineares; $\beta_{11}, \beta_{22}, \beta_{33}$ os coeficientes quadráticos; $\beta_{12}, \beta_{13}, \beta_{23}$ os coeficientes das interações e, $X_{\mathrm{i}} X_{\mathrm{j}}$ os valores codificados para os níveis das variáveis $X_{i} X_{j}$; $\varepsilon$ é o erro aleatório. O programa Statistica versão 7.0 foi utilizado para a análise de variância (ANOVA) e definir o coeficiente de determinação $\left(\mathrm{R}^{2}\right)$.

A separação da biomassa do meio de cultivo foi realizada por floculação seguida de centrifugação. O grau de floculação da suspensão celular foi observado por meio da determinação da relação entre a altura do sedimento (Hs) e a altura da fase líquida (Hl). A relação Hs/Hl indica o volume de sedimentação (VS). Quanto maior for o volume de sedimentação (VS), maior é o grau de floculação da suspensão e mais facilmente é a sua redispersão. ${ }^{21}$ A suspensão celular decantada foi centrifugada a $4000 \mathrm{rpm}$ por 40 minutos sob refrigeração de $10^{\circ} \mathrm{C}$. A biomassa resultante foi mantida em estufa de secagem a $60{ }^{\circ} \mathrm{C}$ por 12 horas e utilizada para análises posteriores.

\section{Métodos de extração e quantificação de lipídeos}

Para a obtenção do extrato lipídico da biomassa de Chlorella sp. foram utilizados três métodos de extração. O método de Soxhlet (extração por solvente) e o método de extração por fluído supercrítico (EFS $-\mathrm{CO}_{2}$ ), com o rompimento prévio da parede celular através da sonicação (ultrassom) em equipamento Ultrasonic Cleaner (Indaiatuba, São Paulo, Brasil) a $20 \mathrm{~Hz}$ durante 30 minutos e a EFS $-\mathrm{CO}_{2}$ sem o processo de sonicação.

\section{Método de Soxhlet}

Foram pesados $50,0 \mathrm{~g}$ da amostra e transferido para o cartucho de extração em aparelho de Soxhlet com $n$-hexano como solvente de extração, durante um período de 4 a 8 horas. A temperatura foi mantida constante no aparelho extrator, dentro da faixa de ebulição do $n$-hexano $\left(68-70{ }^{\circ} \mathrm{C}\right)$. Balões de $250 \mathrm{~mL}$ foram utilizados no aparato e o volume de solvente usado para cada extração foi de aproximadamente $170 \mathrm{~mL}$. Após o término do período de extração e arrefecimento da micela foram retiradas alíquotas de 3,0 $\mathrm{mL}$ para análises de lipídios totais.

\section{Método extração por fluido supercrítico - $\mathrm{CO}_{2}$}

A extração por fluido supercrítico $\left(\mathrm{EFS}-\mathrm{CO}_{2}\right.$ ) foi conduzida em equipamento SFT-100XW (Newark, Delaware, USA) a uma pressão de 4440 psi (306 bar) e temperatura de $60^{\circ} \mathrm{C}$. Pesou-se $10 \mathrm{~g}$ de biomassa seca e cristais de vidro com diâmetro de 3,0 $\mathrm{mm}$ foram misturados e colocados no cartucho de extração de alta pressão. $\mathrm{O}$ $\mathrm{CO}_{2}$ foi pressurizado à pressão de operação através de uma bomba de alta pressão. $\mathrm{O} \mathrm{CO}_{2}$ pressurizado foi aquecido à temperatura de operação e bombeado para dentro do cartucho durante o processo de extração. O extrato lipídico foi coletado em frasco de vidro previamente tarado através do fluxo de $\mathrm{CO}_{2}$ pela amostra regulada pela válvula restritora durante 4 horas de extração. O extrato lipídico no 
frasco foi posteriormente quantificado por gravimetria, obtendo-se assim o extrato lipídico total que foi utilizado para a análise do perfil dos ácidos graxos.

\section{Caracterização dos ácidos graxos}

A transesterificação do extrato lipídico total foi realizada de acordo com o método de Metcalfe. ${ }^{22} \mathrm{~A}$ análise da composição em ácidos graxos dos extratos lipídicos foi determinada por cromatografia gasosa (CG) utilizando cromatógrafo a gás, modelo GC-2014 (Shimadzu, Kyoto, Japão) equipado com detector de ionização de chama e coluna com 105 metros de comprimento e $0,25 \mathrm{~mm}$ de diâmetro interno (10\% cyanopropylphenyl, $90 \%$ biscianopropil polysiloxane), com fluxo de $1,1 \mathrm{~mL} / \mathrm{min}$, temperatura do injetor de $260^{\circ} \mathrm{C}$ e temperatura do detector $260^{\circ} \mathrm{C}$. A composição dos ácidos graxos foi determinada por comparação dos picos do tempo de retenção com os respectivos padrões de ácidos graxos (Sigma, St. Louis, USA).

\section{RESULTADOS E DISCUSSÃO}

Na Figura 1 é possível visualizar a curva de crescimento da microalga Chlorella sp. cultivada em BBM padrão e BBM + concentrado de dessalinização (CD). Foi observado que a densidade celular de Chlorella sp. foi maior no cultivo em BBM padrão em relação ao BBM + CD. Uma possível explicação para a redução da densidade celular no BBM + CD é a elevada concentração de íons divalentes no concentrado de dessalinização. De modo provável, a alta concentração de íons divalentes (cálcio, magnésio e sulfatos) no concentrado de dessalinização pode causar inibição do crescimento microalgal. ${ }^{23}$

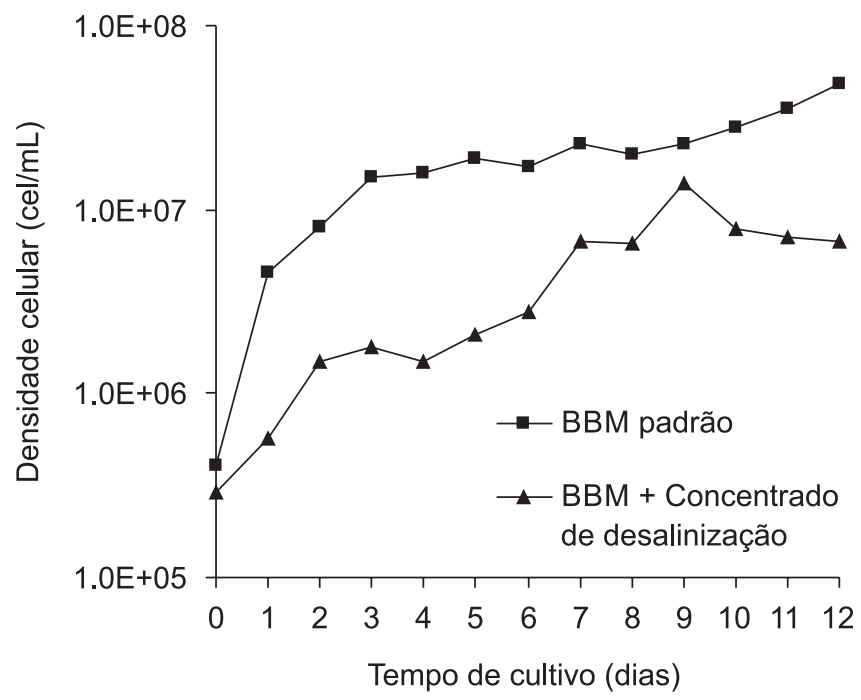

Figura 1. Densidade celular de Chlorella sp. cultivada em meio BBM padrão e $B B M+$ concentrado de dessalinização $(C D)$

\section{Efeitos do delineamento Box-Behnken}

A região experimental estudada foi definida com base em estudos prévios e na literatura. ${ }^{12,14}$ Para avaliar a influência das variáveis independentes - concentração de cloreto de cálcio e o pH - sobre a variável dependente volume de sedimentação (VS), foi elaborado um planejamento estatístico Box-Behnken com a finalidade de determinar a concentração ideal do cloreto de cálcio e a faixa do $\mathrm{pH}$. Os valores da resposta do volume de sedimentação(VS) estão expressos na Tabela 2.

Tabela 2. Matriz de Delineamento Box-Behnken com o volume de sedimentação da biomassa de Chlorella sp.

\begin{tabular}{cccc}
\hline Tratamentos & $\mathrm{CaCl}_{2}(\mathrm{~g} / \mathrm{L})$ & $\mathrm{pH}$ & $\begin{array}{c}\text { Volume de } \\
\text { sedimentação }\end{array}$ \\
\hline 1 & $0,5(-1)$ & $7,0(-1)$ & 0,015 \\
2 & $0,5(-1)$ & $8,0(0)$ & 0,038 \\
3 & $0,5(-1)$ & $9,0(+1)$ & 0,085 \\
4 & $1,5(0)$ & $7,0(-1)$ & 0,046 \\
5 & $1,5(0)$ & $8,0(0)$ & 0,062 \\
6 & $1,5(0)$ & $9,0(+1)$ & 0,054 \\
7 & $2,5(+1)$ & $7,0(-1)$ & 0,046 \\
8 & $2,5(+1)$ & $8,0(0)$ & 0,069 \\
9 & $2,5(+1)$ & $9,0(+1)$ & 0,038 \\
10 & $1,5(0)$ & $8,0(0)$ & 0,054 \\
11 & $1,5(0)$ & $8,0(0)$ & 0,046 \\
\hline 12 & $1,5(0)$ & $8,0(0)$ & 0,054 \\
\hline
\end{tabular}

O modelo matemático empírico, codificado e de $2^{\mathrm{a}}$ ordem, encontrado, com os seus respectivos coeficientes estatísticos está apresentado na Equação 2. Esse modelo foi obtido a partir da regressão não linear (modelo quadrático) dos dados experimentais, utilizando o software Statistica versão 7.0

$\mathrm{VS}=0,608-0,041 \mathrm{X}_{1}-0,342 \mathrm{X}_{1}^{2}-0,203 \mathrm{X}_{2}+0,016 \mathrm{X}_{2}^{2}+$ $0,007 \mathrm{X}_{1} \mathrm{X}_{2}$

O resultado da análise de variância (ANOVA) para a resposta volume de sedimento (VS) está apresentado na Tabela 3, na qual se observa que o coeficiente de determinação $\mathrm{R}^{2}$ foi de 0,96 mostrando que $96,0 \%$ da variação da resposta pode ser explicada pelo modelo. $\mathrm{O}$ volume de sedimentação da biomassa variou de 0,015 a 0,085 . Houve efeito significativo $(\mathrm{p}<0,05)$ para variável $\mathrm{pH}$ e a interação entre as variáveis, sugerindo que a concentração de cloreto de cálcio e o pH do meio influenciaram na resposta. $\mathrm{O}$ tratamento 3 apresentou o maior volume de sedimentação da biomassa $(0,085)$ com valores fixados de $0,5 \mathrm{~g} / \mathrm{L}$ de cloreto de cálcio e pH 9,0. A análise de variância dos resultados do delineamento de Box-Behnken para a resposta avaliada,

Tabela 3. Análise de Variância (ANOVA) após o Delineamento Box-Behnken para a variável resposta volume de sedimentação da biomassa de Chlorella sp.

\begin{tabular}{lccccc}
\hline & Soma quadrática & Grau de liberdade & Média quadrática & Teste F & $\mathrm{p}$ \\
\hline $\mathrm{CaCl}_{2}(\mathrm{~L}+\mathrm{Q})$ & 0,000056 & 2 & 0,000028 & 0,69870 & 0,563493 \\
$\mathrm{pH}(\mathrm{L}+\mathrm{Q})$ & 0,000915 & 2 & 0,000457 & 11,42482 & $0,039537^{*}$ \\
Interação $(\mathrm{L}+\mathrm{Q})$ & 0,002192 & 4 & 0,000548 & 13,68802 & $0,028676^{*}$ \\
Erro puro & 0,000120 & 3 & 0,000040 & - & - \\
\hline
\end{tabular}

\begin{tabular}{lcc}
\hline Total SS & 0,003302 & 11 \\
$\mathrm{R}^{2}=0,96 ; \mathrm{R}_{\mathrm{adj}}=0,86 ; * \mathrm{p}<0,05 ; \mathrm{L}=$ efeito linear; $\mathrm{Q}=$ efeito quadrático.
\end{tabular}


volume de sedimentação da biomassa, indicou que a regressão não foi significativa ao nível de $5 \%$. A falta de ajuste também não foi significativa, o que permite a utilização do modelo matemático.

Baseado nos resultados obtidos pelo delineamento de BoxBenhken foi realizado um delineamento Central Composto (DCC) a fim de se obter uma melhor condição para a floculação da biomassa de Chlorella sp.

\section{Otimização do volume de sedimentação pelo Delineamento Central Composto (DCC)}

A Tabela 4 mostra o delineamento estatístico central composto utilizado como experimento de ajuste para a determinação da melhor condição de obtenção do volume de sedimentação da biomassa microalgal. O modelo matemático polinomial de $2^{\mathrm{a}}$ ordem para o volume de sedimentação (VS) está descrito na Equação 3:

$$
\mathrm{VS}=-1,256-0,124 \mathrm{X}_{1}-0,010 \mathrm{X}_{1}^{2}+0,286 \mathrm{X}_{2}-0,015 \mathrm{X}_{2}^{2}+
$$
$0,018 \mathrm{X}_{1} \mathrm{X}_{2}$

Na Tabela 4 é possível visualizar que o volume de sedimentação da biomassa variou de 0,044 a 0,147 . O tratamento 10 apresentou o melhor resultado experimental $(0,147)$ do volume de sedimentação, no qual a concentração de $\mathrm{CaCl}_{2}$ foi de $2,0 \mathrm{~g} / \mathrm{L}$ e o pH igual a 9,5. A análise estatística (ANOVA) (Tabela 5) das variáveis independentes mostrou que a variável $\mathrm{pH}$ apresentou efeito significativo. O coeficiente de determinação $\mathrm{R}^{2}$ para o modelo foi de 0,85 mostrando que $85,0 \%$ da variação da resposta pode ser explicada pelo modelo, sendo este valor aceitável quando se trata de sistemas biológicos. A partir da avaliação do modelo quadrático obtido para a variável volume de sedimentação (VS), foi possível gerar as curvas de contorno da influência da concentração de cloreto de cálcio e do $\mathrm{pH}$ sobre o volume de sedimentação (Figura 2).

Tabela 4. Matriz do Delineamento Central Composto (DCC) com o volume de sedimentação da biomassa de Chlorella sp.

\begin{tabular}{cccc}
\hline Tratamentos & $\mathrm{CaCl}_{2}(\mathrm{~g} / \mathrm{L})$ & $\mathrm{pH}$ & $\begin{array}{c}\text { Volume de } \\
\text { sedimentação }\end{array}$ \\
\hline 1 & $1,0(-1)$ & $8,5(-1)$ & 0,073 \\
2 & $1,0(-1)$ & $10,5(+1)$ & 0,088 \\
3 & $3,0(+1)$ & $8,5(-1)$ & 0,044 \\
4 & $3,0(+1)$ & $10,5(+1)$ & 0,132 \\
5 & $0,6(-\alpha)$ & $9,5(0)$ & 0,088 \\
6 & $3,4(+\alpha)$ & $9,5(0)$ & 0,117 \\
7 & $2,0(0)$ & $8,0(-\alpha)$ & 0,044 \\
8 & $2,0(0)$ & $11,0(+\alpha)$ & 0,132 \\
9 & $2,0(0)$ & $9,5(0)$ & 0,102 \\
10 & $2,0(0)$ & $9,5(0)$ & 0,147 \\
11 & $2,0(0)$ & $9,5(0)$ & 0,102 \\
12 & $2,0(0)$ & $9,5(0)$ & 0,117 \\
13 & $2,0(0)$ & $9,5(0)$ & 0,117 \\
\hline
\end{tabular}

Foi observado que o volume de sedimentação (VS) é máximo quando a concentração do cloreto de cálcio for fixada em $2,0 \mathrm{~g} / \mathrm{L}$ e o valor de $\mathrm{pH}$ entre 9,5 e 11,5. Na Figura 3 é possível visualizar claramente a floculação da cultura de Chlorella sp. após a otimização. Em comparação com a literatura, os valores de $\mathrm{pH}$ obtidos neste estudo são semelhantes ao demonstrado por Vandamme et $a l .{ }^{24}$ na qual a floculação de microalga Chlorella vulgaris foi induzida em
Tabela 5. Análise de Variância (ANOVA) após o Delineamento Central Composto (DCC) para a variável resposta volume de sedimentação da biomassa de Chlorella sp.

\begin{tabular}{lccccc}
\hline & $\begin{array}{c}\text { Soma } \\
\text { quadrática }\end{array}$ & $\begin{array}{c}\text { Grau de } \\
\text { liberdade }\end{array}$ & $\begin{array}{c}\text { Média } \\
\text { quadrática }\end{array}$ & Teste F & $\mathrm{p}$ \\
\hline $\mathrm{CaCl}_{2}(\mathrm{~L})$ & 0,000394 & 1 & 0,000394 & 1,20999 & 0,333085 \\
$\mathrm{CaCl}_{2}(\mathrm{Q})$ & 0,000779 & 1 & 0,000779 & 2,39249 & 0,196824 \\
$\mathrm{pH}(\mathrm{L})$ & 0,006508 & 1 & 0,006508 & 19,98766 & $0,011068^{*}$ \\
$\mathrm{pH}(\mathrm{Q})$ & 0,002022 & 1 & 0,002022 & 6,21017 & 0,067339 \\
$\begin{array}{l}\text { Interação } \\
(\mathrm{L}+\mathrm{Q})\end{array}$ & 0,001351 & 1 & 0,001351 & 4,14783 & 0,111371 \\
$\begin{array}{l}\text { Falta de } \\
\text { ajuste }\end{array}$ & 0,000447 & 3 & 0,000149 & 0,45798 & 0,726446 \\
Erro puro & 0,001302 & 4 & 0,000326 & - & - \\
\hline Total SS & 0,012476 & 12 & - & - & - \\
\hline $\mathrm{R}^{2}=0,85 ; \mathrm{R}_{\text {adj }}=0,75 ; * \mathrm{p}<0,05 ; \mathrm{L}=$ efeito linear; $\mathrm{Q}=$ efeito quadrático.
\end{tabular}

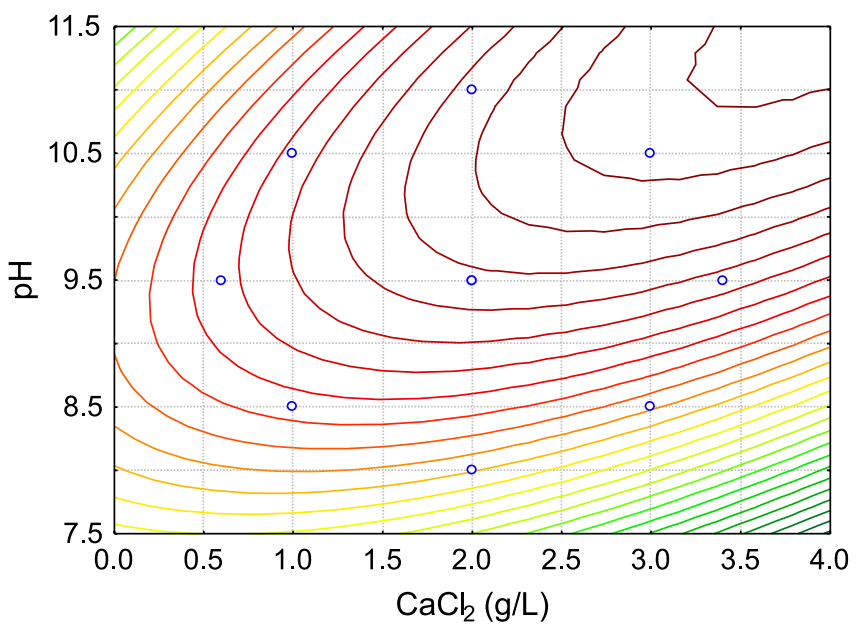

Figura 2. Gráfico de contorno do Delineamento Central Composto (DCC) em função da concentração de $\mathrm{CaCl}_{2}(\mathrm{~g} / \mathrm{L})$ e $\mathrm{pH}$

pH entre 11,0 - 12,0. O mesmo autor considera que a floculação pode ocorrer naturalmente quando o suprimento de $\mathrm{CO}_{2}$ é interrompido e, devido ao processo fotossintético, o $\mathrm{pH}$ é elevado a próximo de 10,0. Neste estudo, para que ocorra uma eficiente floculação da biomassa de Chlorella sp. foi necessário adicionar aproximadamente 2,0 g/L de cloreto de cálcio ao meio de cultivo. A presença de sais de cálcio e/ou magnésio, que apresentam caráter básico, favorece a formação de flocos microalgais nos quais a carga positiva do cloreto de cálcio neutraliza a carga negativa presente na superfície das microalgas, induzindo a floculação e agregação das células. ${ }^{12}$ Ainda, é de notar que os íons divalentes (cálcio e magnésio) estão presentes no concentrado de dessalinização, podendo induzir a floculação. No entanto, a baixa concentração destes sais no concentrado de dessalinização não irá favorecer naturalmente o fenômeno da floculação. De fato, Nurdogan e Oswald ${ }^{25}$ observaram em tanques abertos de cultivo de microalgas que a autofloculação não ocorre em águas com baixo teor de cálcio e magnésio. Desta maneira, a escolha do cloreto de cálcio como agente floculante deve ser destacada.

\section{Métodos de extração e perfil dos ácidos graxos}

Três diferentes métodos de extração dos lipídeos da biomassa de Chlorella sp. foram propostos neste estudo. O teor de lipídeos foi maior pelo método de Soxhlet $(23,0 \%)$, seguido por extração 


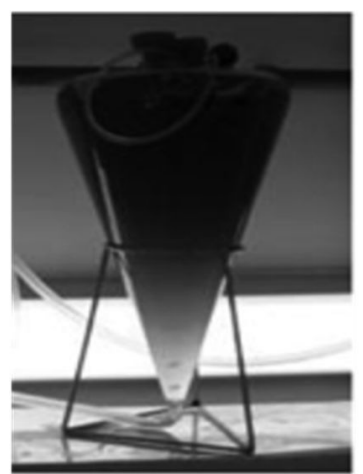

Cultura de Chlorella sp.

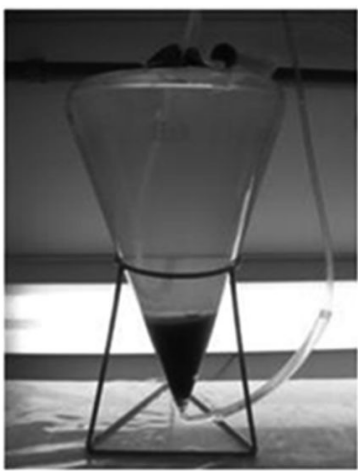

Cultura de Chlorella sp. floculada
Figura 3. Floculação da cultura de Chlorella sp. após a otimização, condição da floculação: $\mathrm{CaCl}(2,0 \mathrm{~g} / \mathrm{L})$ e pH $=10,0$ - 11,0

por fluído supercrítico (EFS) com sonicação (4,5\%) e EFS sem sonicação $(2,1 \%)$. O perfil dos ácidos graxos extraídos da biomassa de Chlorella sp. em três diferentes métodos de extração está apresentado na Tabela 6. Nos três métodos de extração testados o ácido palmítico (C16:0) foi o ácido graxo observado em maior concentração, sendo maior no método de Soxhlet (57,0\%), seguido da EFS com sonicação $(35,3 \%)$ e da EFS sem sonicação $(25,5 \%)$. Também houve a produção do ácido linoléico $(\mathrm{C} 18: 2 \mathrm{n} 6 \mathrm{c})$ em todas as condições experimentadas. A biomassa obtida do cultivo da microalga Chlorella sp. apresentou elevado teor de ácido linolênico $(\mathrm{C} 18: 3 \mathrm{n} 3 \mathrm{c})$ com $10,9 \%$ pelo método de Soxhlet e 24,5\% pela EFS com sonicação. Diversos ácidos graxos não foram possíveis de ser detectados na EFS sem sonicação. Neto et $a l .{ }^{26}$ sugere que a extração dos lipídeos de microalgas pelo método de

Tabela 6. Perfil dos ácidos graxos extraído da biomassa de Chlorella sp. em três diferentes métodos de extração

\begin{tabular}{|c|c|c|c|c|}
\hline \multirow{2}{*}{ Ácidos graxos } & & \multicolumn{3}{|c|}{$\begin{array}{c}\text { Método de extração (valores em } \\
\text { porcentagem) }\end{array}$} \\
\hline & & Soxhlet & $\begin{array}{l}\text { EFS (sem } \\
\text { sonicação) }\end{array}$ & $\begin{array}{l}\text { EFS (com } \\
\text { sonicação) }\end{array}$ \\
\hline \multirow[t]{7}{*}{ Saturados } & C13:0 & 0,38 & nd & nd \\
\hline & $\mathrm{C} 14: 0$ & 1,47 & nd & 0,82 \\
\hline & $\mathrm{C} 15: 0$ & 1,30 & nd & 0,84 \\
\hline & C16:0 & 57,04 & 25,52 & 35,36 \\
\hline & $\mathrm{C} 17: 0$ & 0,75 & 0,44 & 0,42 \\
\hline & C18:0 & 2,65 & 3,02 & 2,06 \\
\hline & Subtotal & 63,59 & 28,98 & 39,50 \\
\hline \multirow[t]{3}{*}{ Monoinsaturados } & C15:1 & 2,47 & 1,21 & 8,96 \\
\hline & C16:1 & 1,05 & 1,87 & 1,53 \\
\hline & Subtotal & 3,52 & 3,08 & 10,49 \\
\hline \multirow[t]{7}{*}{ Poli-insaturados } & $\mathrm{C} 18: 2 \mathrm{n} 6 \mathrm{c}$ & 4,97 & 8,49 & 7,67 \\
\hline & C18:3n3 & 10,99 & nd & 24,51 \\
\hline & $\mathrm{C} 18: 1 \mathrm{n} 9 \mathrm{c}$ & nd & 0,36 & 1,38 \\
\hline & $\mathrm{C} 20: 2$ & nd & nd & 0,44 \\
\hline & Subtotal & 15,96 & 8,85 & 34,00 \\
\hline & $\begin{array}{l}\text { Não identi- } \\
\text { ficados }\end{array}$ & 16,93 & 59,09 & 16,01 \\
\hline & Total & 100 & 100 & 100 \\
\hline Lipídeos totais & & 23,3 & 2,1 & 4,5 \\
\hline
\end{tabular}

EFS: Extração por Fluído Supercrítico.
EFS pode ser acompanhada de um pré-tratamento de ruptura celular (sonicação), ou ainda, conduzido com um co-solvente de extração. Também, a moagem e trituração, acompanhadas de um pré-tratamento enzimático ou micro-ondas, são ferramentas potenciais que podem otimizar a biodisponibilidade celular de lipídeos. ${ }^{2}$

É importante salientar que devido à espessura da parede celular das microalgas do gênero Chlorella, a liberação dos lipídeos intracelulares fica bloqueada. Portanto, o emprego de um pré-tratamento por sonicação facilita a extração lipídica. Logo, foi verificado que a detecção dos ácidos graxos foi mais eficiente no processo de EFS (com sonicação). Ainda, dentre os três métodos de extração de lipídeos proposto, o maior teor de ácidos graxos poli-insaturados $(34,0 \%)$ foi alcançado na EFS (com sonicação). Ademais, estes compostos extraídos pelo processo de EFS não sofrem a biodegradação pelos solventes (método de Soxhlet), tornando adequados para a incorporação em alimentos funcionais. Apesar da EFS ser um método inovador, a eficiência na extração dos lipídeos não foi equivalente ao método convencional de extração por solventes (Soxhlet). Por conseguinte, a utilização da EFS- $\mathrm{CO}_{2}$ na extração de carotenóides de microalgas como corantes naturais seria uma alternativa viável para a aplicação na indústria de alimentos. ${ }^{27}$

Em resumo, este estudo revelou que se a intenção dos lipídeos obtidos a partir do cultivo da microalga Chlorella sp. em concentrado de dessalinização for para a conversão do óleo em biodiesel recomenda-se o método de extração por Soxhlet. Por outro lado, a EFS- $\mathrm{CO}_{2}$ seria o método de escolha quando os lipídeos e ácidos graxos fossem aplicados na indústria alimentícia.

\section{CONCLUSÕES}

O modelo matemático obtido pela metodologia de superfície de resposta indicou que a concentração de cloreto de cálcio fixada em 2,0 g/L e pH 10,0 são as condições mais adequadas para a floculação da biomassa de Chlorella sp. e, portanto, um método eficiente para pré-concentrar a cultura antes do processo de centrifugação.

A eficiência da extração de lipídeos da biomassa de Chlorella sp. foi diferente de acordo com o método de extração. O maior teor lipídico foi obtido pelo método de Soxhlet 23,3\%, seguido da EFS com sonicação com $4,5 \%$ e da EFS sem sonicação $2,1 \%$. O ácido palmítico (C16:0) foi o ácido graxo predominante nos três métodos de extração. A microalga Chlorella sp. exibiu elevado teor de ácidos graxos poli-insaturados, tais como os ácidos linolênico e linoléico.

Por fim, o concentrado de dessalinização é fonte de nutrientes prontamente disponível para o cultivo de Chlorella sp. Portanto, representa uma opção atrativa para o desenvolvimento de complementos nutricionais (health foods), ração animal suplementada e potencial para a produção de biocombustíveis em virtude do alto teor de lipídeos e perfil dos ácidos graxos.

\section{AGRADECIMENTOS}

Os autores agradecem ao suporte financeiro pelo Conselho Nacional de Desenvolvimento Científico e Tecnológico (CNPq) (574.716/2008-4) e a CAPES pela concessão da bolsa de estudos.

\section{REFERÊNCIAS}

1. Carioca, J. O. Em Química verde no Brasil: 2010 - 2030; Carioca, J. O.; Almeida, M. F.; Seidl, P. R., eds.; Centro de Gestão e Estudos Estratégicos: Brasília, 2010, cap. 1.

2. Pereira, C. M. P.; Hobuss, C. B.; Maciel, J. V.; Ferreira, L. R.; Del Pino, F. B.; Mesko, M. F.; Quim. Nova 2012, 35, 2013.

3. Biller, P.; Ross, A. B.; Skill, S. C.; Lea-Langton, A.; Balasundaram, B.; Hall, C.; Riley, R.; Llewellyn, C. A.; Algal Research 2012, 1, 70. 
4. Volkmann, H.; Imianovsky, U.; Oliveira, J. L. B.; Sant'Anna, E. S.; Braz. J. Microb. 2008, 39, 98.

5. Pittman, J. K.; Dean, A. P.; Osudenko, O.; Bioresour. Technol. 2011, 102, 17.

6. Menezes, J. S.; Campos, V. P.; Costa, T. A. C.; Desalination 2011, 281, 271.

7. Li, Y.; Chen, Y. F.; Chen, P.; Min, M.; Zhou, W.; Martinez, B.; Zhu, J.; Ruan, R.; Bioresour. Technol. 2011, 102, 5138.

8. Matos, A. P. Dissertação de Mestrado, Universidade Federal de Santa Catarina, Brasil, 2012.

9. Conceição, R. C.; Frasão, C. V.; Silva, S. M. C.; Medeiros, J. L.; Araújo, O. Q. F.; Picardo, M. C.; Quim. Nova 2012, 35, 1336.

10. Uduman, N.; Qi Y.; Danquah, M. K.; Forde, G. M.; Hoadley, A.; J. Renewable Sustainable Energy 2010, 2, 2701.

11. Mata, T. M.; Martins, A. A.; Caetano, N.; Renewable Sustainable Energy Rev. 2010, 14, 217.

12. Smith, B. T.; Davis, R. H.; Algal Research 2012, 1, 32.

13. Molina, G. E.; Belari, E.; Acien, F. F. G.; Robles, M. A.; Chisty, Y.; Biotechnol. Adv. 2003, 20, 491.

14. Yang, Z. H.; Huang, J.; Zeng, G. M.; Ruan, M.; Zhou, C. S.; Li, L.; Rong, Z. G.; Bioresour. Technol. 2009, 100, 4233.

15. Lee, J. Y.; Yoo, C.; Jun, S. Y.; Ahn, C. Y.; Oh, H. M.; Bioresour. Technol. 2010, 101, 75.

16. Cheng, C. H.; Du, T. B.; Pi, H. C.; Jang, S. M.; Lin, Y. H.; Lee, H. T.; Bioresour. Technol. 2011, 102, 10151.
17. Sahena, F.; Zaidul, I. S. M.; Jinap, S.; Karim, A. A.; Abbas, K. A.; Norulaini, N. A. N.; Omar, K. M.; J. Food Eng. 2009, 95, 240.

18. American Public Health Association. Standard methods for the examination of water and wastewater, 20 $0^{\text {th }}$ ed., APHA: New York, 1998.

19. Nichols, H.W. In Handbook of Phycological Methods: Culture Methods and Growth Measurements; Stein, J., ed.; Cambridge University Press: Cambridge, 1973, pp. 7-24.

20. Box, G. E. P.; Behnken, D. W. Technometrics 1961, 2, 455

21. Prista, L. N.; Alves, A. C.; Morgado, R. M. R.; Tecnologia Farmacêutica v. 1, $5^{\text {a }}$ ed., Fundação Calouste Gulbenkian: Lisboa, 1995, p. 199-478.

22. Metcalfe, L. D.; Wang, C. N.; J. Chromatogr. Sci. 1981, 19, 530.

23. Myint, M. T.; Hussein, W. New Mexico State University, Institute of Energy \& the Environment, 2012. http://www.ieenmsu.com/2012/05/04/ microalgae-strain-could-reduce-algae-and-desalination-costs/. Acessado em Agosto 2013.

24. Vandamme, D.; Foubert, I.; Fraeye, I.; Meesschaert, B.; Muylaert, K.; Bioresour. Technol. 2012, 105, 114.

25. Nurdogan, Y.; Oswald, W. J.; Water Sci. Technol. 1995, 31, 33.

26. Neto, A. M. P.; Souza, R. A. S.; Leon-Nino, A. D.; Costa, J. D. A.; Tiburcio, R. S.; Nunes, T. A.; Mello, T. C. S.; Kanemoto, F. T.; Saldanha-Corrêa, F. M. P.; Gianesella, S. M. F.; Renewable Energy 2013, 55,525

27. Macías-Sánches, M. D.; Mantell, C.; Rodríguez, M.; Martínez de la Ossa, E.; Lubían, L. M.; Montero, O.; J. Food Eng. 2005, 66, 245. 